\title{
Three-Dimensional Modeling and Diversity Analysis Reveals Distinct AVR Recognition Sites and Evolutionary Pathways in Wild and Domesticated Wheat Pm3 R Genes
}

\author{
Hanan Sela, ${ }^{1,2}$ Laurentiu N. Spiridon, ${ }^{3}$ Haim Ashkenazi, ${ }^{1}$ Navreet K. Bhullar, ${ }^{4}$ Susanne Brunner, ${ }^{4}$ \\ Andrei-Jose Petrescu, ${ }^{3}$ Tzion Fahima, ${ }^{1}$ Beat Keller, ${ }^{4}$ and Tina Jordan ${ }^{4}$ \\ ${ }^{1}$ Department of Evolutionary and Environmental Biology, Institute of Evolution, Faculty of Science and Science Education, \\ University of Haifa, Mt. Carmel, Haifa, 31905 Israel; ${ }^{2}$ The Institute for Cereal Crops Improvement, Tel-Aviv University P.O. \\ Box 39040, Tel Aviv 69978 Israel; ${ }^{3}$ Institute of Biochemistry of the Romanian Academy, Splaiul Independentei 296, 060031 \\ Bucharest 17, Romania; ${ }^{4}$ Institute of Plant Biology, University of Zürich, Zollikerstrasse 107, 8008 Zürich, Switzerland
}

Submitted 15 January 2014. Accepted 7 April 2014.

\begin{abstract}
The $P m 3$ gene confers resistance against wheat powdery mildew. Studies of $P m 3$ diversity have shown that $P m 3$ alleles isolated from southern populations of wild emmer wheat located in Lebanon, Jordan, Israel, and Syria are more diverse and more distant from bread wheat alleles than alleles from the northern wild wheat populations located in Turkey, Iran, and Iraq. Therefore, southern populations from Israel were studied extensively to reveal novel Pm3 alleles that are absent from the cultivated gene pool. Candidate $P m 3$ genes were isolated via a polymerase chain reaction cloning approach. Known and newly identified $P m 3$ genes were subjected to variation analysis and polymorphic amino acid residues were superimposed on a three-dimensional (3D) model of PM3. The region of highest interspecies diversity between Triticum aestivum and $T$. dicoccoides lies in leucine-rich repeats (LRR) 19 to 24, whereas most intraspecies diversity in T. aestivum is located in LRR 25 to 28 . Interestingly, these two regions are separated by one large LRR whose propensity for flexibility facilitates the conformation of the PM3 LRR domain into two differently structured models. The combination of evolutionary and protein 3D structure analysis revealed that $P m 3$ genes in wild and domesticated wheat show different evolutionary histories which might have been triggered through different interactions with the powdery mildew pathogen.
\end{abstract}

H. Sela and L. N. Spiridon contributed equally to the work.

Current address for N. K. Bhullar: Department of Biology, Plant Biotechnology, Swiss Federal Institute of Technology, Universitätsstrasse 2, 8092 Zurich, Switzerland.

Sequences were deposited in the National Center for Biotechnology Information GenBank under accession numbers JF739306 to JF739353.

Corresponding authors: H. Sela; E-mail: hans@tauex.tau.ac.il; and T. Jordan; E-mail: tjordan@botinst.uzh.ch

* The $\boldsymbol{e}$-Xtra logo stands for "electronic extra" and indicates that two supplementary figures and one supplementary table are published online and that Figure 2 appears in color online.

(C) 2014 The American Phytopathological Society
The coevolution of plants and their pathogens shaped a multilayered immune system in plants (Dangl and Jones 2001). The majority of intracellular immune receptors (so-called $\mathrm{R}$ proteins) form modular nucleotide-binding (NB-ARC) leucinerich repeat (LRR) proteins. Structure-function analysis of these proteins revealed that the NB-ARC domain works as a reversible molecular switch to control protein activity (Takken and Tameling 2009; Takken et al. 2006). Typically, each repeat of the LRR domain contains the conserved consensus motif LxxLxLxxN(Cx)xL (Wei et al. 2008) and is 20 to 29 residues long. The repeats form $\beta$-strand or $\beta$-turn structures in which the variable nonleucine residues shape the concave, solventexposed surface of the horseshoe-like structure. The individual LRR of plants are highly irregular, with varying lengths and noncanonical LRR motifs. The dual function of the LRR domain is i) to keep the protein in an auto-inhibited state through interaction with the NB-ARC domain (Takken et al. 2006) and ii) to confer pathogen recognition specificity. The importance of polymorphic amino acids in the LRR domain for recognition specificity was shown in a number of studies (Brunner et al. 2010; Dodds et al. 2001; Kanzaki et al. 2012; Krasileva et al. 2010). The coevolution of pathogen and host results in changes of recognition specificity, which can be observed in the form of diversifying selection within the LRR region. Such diversifying selection has been suggested to indicate a direct LRR-avirulence protein (AVR) interaction. Indeed, for $L$ (Dodds et al. 2006), Pik (Kanzaki et al. 2012), and RPPl (Krasileva et al. 2010) resistance genes, it was shown that their products interact directly with their cognate AVR proteins.

To our knowledge, no plant R protein LRR crystal structures have been generated until now. This impedes the structural analysis of AVR-LRR interaction or recognition. Remote homology modeling (Sela et al. 2012; Slootweg et al. 2013) allows the construction of composite models of the LRR domains and can be used as a tool to perform structure-informed and evolutionary studies.

The multi-allelic Pm3 locus from hexaploid wheat confers race-specific resistance to wheat powdery mildew (Blumeria graminis f. sp. tritici). In the modern bread wheat pool, it occurs in seven functionally different alleles, Pm3a to Pm3g. Alleles $P m 3 k$ to $P m 3 t$ were isolated from wild tetraploid wheat and hexaploid landraces (Bhullar et al. 2009, 2010; Yahiaoui et al. 2009). The high sequence conservation of bread wheat Pm3 
alleles suggested their recent evolution from the ancestral sequence Pm3 'ChineseSpring' (CS) after domestication 10,000 years ago. Polymorphic blocks in $P m 3$ sequences derived from tetraploid wheat are different from the blocks found in functional Pm3 alleles of hexaploid wheat, indicating an independent evolution of the Pm3 loci in the two species (Yahiaoui et al. 2009). In PM3, an NB-LRR resistance protein, there is evidence for a major role of the C-terminal LRR in recognition specificity. Previous experiments revealed that PM3E and PM3D specificity is determined exclusively by putative solventexposed residues of the LxxLxLxx motif in the C-terminal LRR (Brunner et al. 2010; Yahiaoui et al. 2006).

Wild emmer wheat (Triticum dicoccoides) is the progenitor of most cultivated wheat species. Its natural habitats are located in the Fertile Crescent of the Middle East. The center of diversity of wild emmer wheat is found in northern Israel and its vicinity (Luo et al. 2007; Nevo and Beiles 1989). Wild emmer populations can be divided into northern (Turkey, Iran, and Iraq) and southern (Lebanon, Jordan, Israel, and Syria) populations. It is widely accepted that domesticated wheat originated from the northern populations in southeast Turkey (Ozkan et al. 2005). Studies of the $P m 3$ diversity have shown that alleles from the southern populations are more diverse and are more distant from the bread wheat alleles than alleles from the northern populations (Yahiaoui et al. 2009). Therefore, in this study, the southern populations from Israel were studied more extensively to reveal novel alleles not present in the cultivated gene pool. Previous studies on the wild emmer population from Israel revealed that this population is highly structured

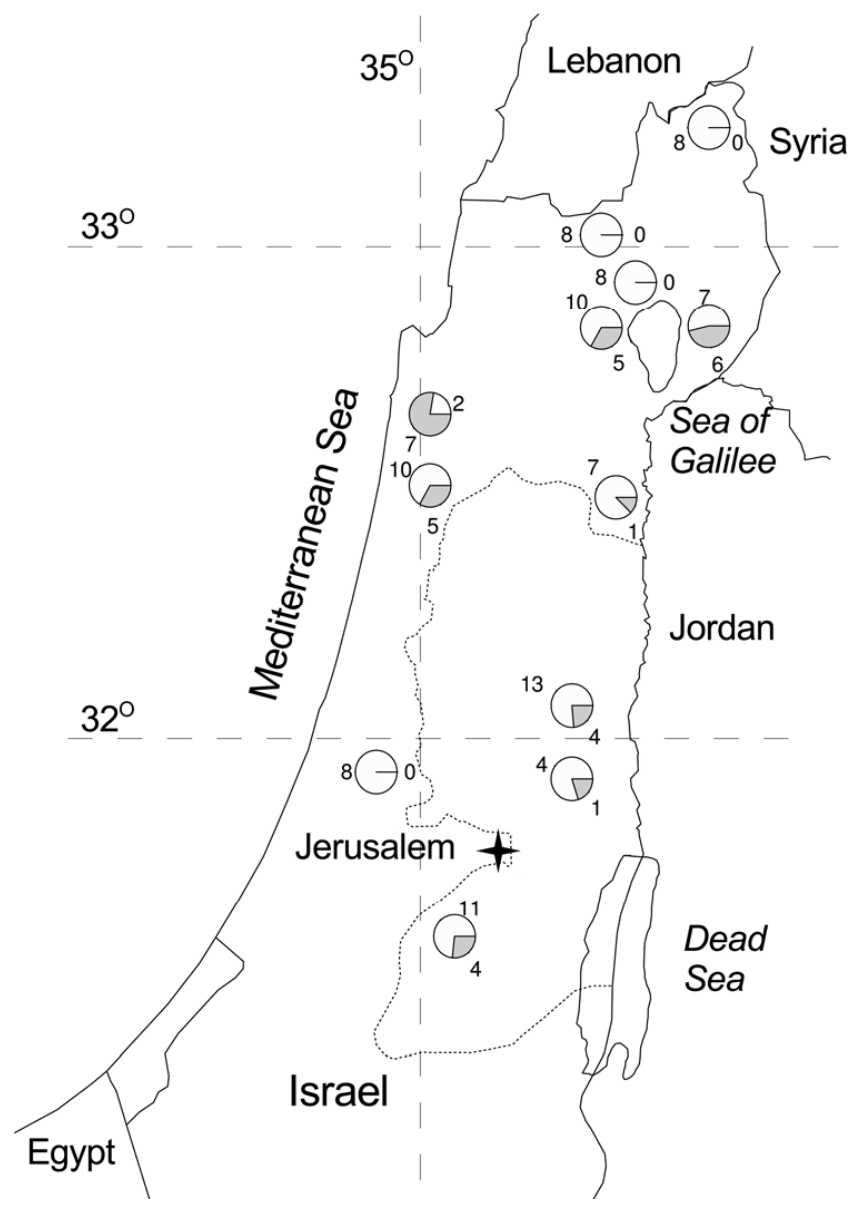

Fig. 1. Geographic distribution of $P m 3$ in Israel as determined by the $P m 3$ haplotype sequence-tagged site marker. The frequency of $P m 3$ in each population is indicated by a pie chart. Positive for $P m 3$ haplotype = gray; negative for $\operatorname{Pm} 3$ haplotype $=$ white . and diverse (Nevo et al. 2002; Peleg et al. 2008). The diversity was found to be partly correlated with climatic factors. The large set of alleles in bread wheat and the presence of $P m 3$ in both wild and domesticated forms of wheat make it an excellent model for evolutionary studies. The objective of the current study was to search for regions and codons that are subjected to selection and to determine their putative significance for AVR recognition and to the overall protein properties, such as stability and structure. Therefore, a three-dimensional (3D) model for PM3 was constructed and was used for superimposing the variation in the amino acid sequence of the LRR domain on the 3D model. Moreover, this study presents the patterns of geographic distribution of $P m 3$ genes in wild emmer wheat populations in Israel. The obtained results will help to design a more efficient conservation and utilization plan of wild emmer important gene pool.

\section{RESULTS}

Isolation of Pm3 genes from wild emmer wheat.

Of the $208 T$. dicoccoides accessions that were used to isolate $P m 3$ genes, 100 were tested for powdery mildew resistance against five different recent Swiss isolates. The majority (79\%) of accessions were resistant to at least one of these isolates. Out of $208 T$. dicoccoides accessions, 81 (39\%) were positive for a Pm3 sequence-tagged site (STS) marker characteristic for the $P m 3$ haplotype, obtained from studies at the Pm3 locus (Srichumpa et al. 2005; Yahiaoui et al. 2004). Pm3 was not present in accessions from Mt. Hermon, the high elevations of upper Galilee, and the Golan Heights whereas, in populations from low elevations, accessions with and without Pm3 haplotype were found (Fig. 1). In 49 (24\%) of all the accessions tested, Pm3-like full-length genes were amplified and sequenced. Sequence alignments revealed that four sequences are paralogs of $P m 3$ based on their higher similarity to already identified $P m 3$ paralogs than to $P m 3$ alleles (Yahiaoui et al. 2004). Consequently, they were named Pm3TD_RGL (resistance gene-like) followed by their accession number: $P m 3 T D_{-}$ RGL255,Pm3TD_RGL_407,Pm3TD_RGL16-40, and Pm3TD_ $R G L 30-8$. The remaining 45 sequences represented 13 different alleles, of which Pm3TDb, Pm3TDd-e, Pm3TDh-j, and Pm3TDm have not been described before (Supplementary Table S1). The distribution of the alleles was not even: allele Pm3TDa was present in 14 of the 45 accessions, allele Pm3TDb was present in 7 accessions, while other alleles were present in only 1 accession, each. In all, 5 to 16 accessions per site were sampled in the 12 collection sites representing T. dicoccoides accessions collected in the 1980s. In eight of these sites, $P m 3$ was present only in some accessions, with a presence/absence ratio of $P m 3$ in each site of 0.25 to 3.5 (average 0.8); and, in four sites, Pm3 was completely absent (Fig. 1).

\section{Geographic distribution of $P m 3$ genes.}

The topology of the maximum likelihood tree of all genotypes consists of a series of three clusters where each cluster stems from the previous cluster (Fig. 2). The order of the clusters is as follows. i) Pm3 alleles originating from the $T$. dicoccoides judaicum race. ii) $T$. dicoccoides alleles from the huranum race (Poyarkova et al. 1991). Within this cluster, there is a subcluster of accessions from the northern populations of Iran and Turkey. iii) This is the most diverse cluster, the cluster of T. aestivum Pm3 alleles. Within the T. aestivum cluster, there are two alleles from wild emmer originating from Turkey, another wild allele from Israel, and three wild emmer accessions from Bat-Shlomo population identical to the Pm3CS allele, which is considered to be the consensus allele of all domesticated wheat alleles (Yahiaoui et al. 2006). It is interesting to 


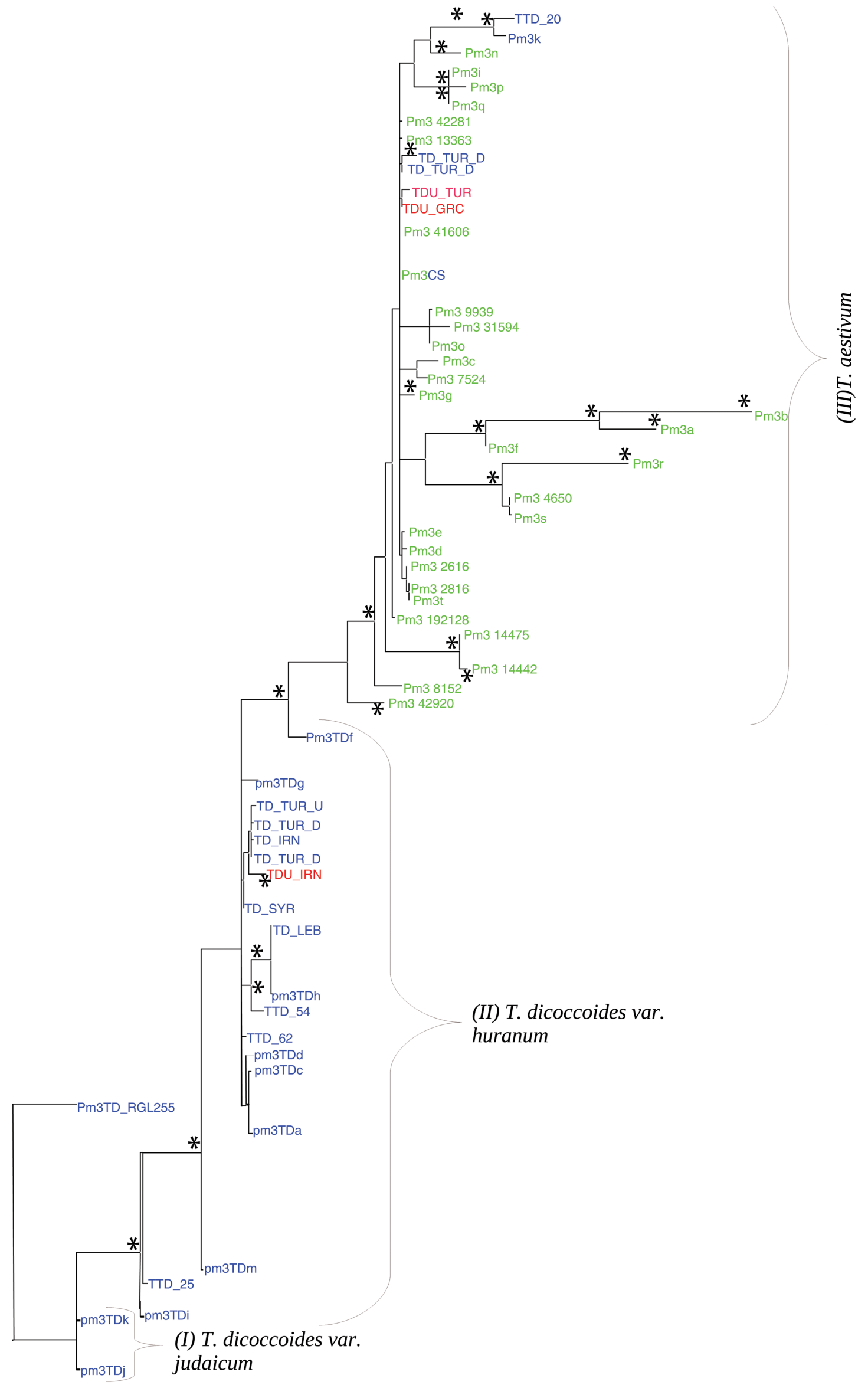

Fig. 2. Maximum likelihood tree of $P m 3$ sequences. Asterisks = branches with signatures of episodic diversifying selection. Numbers I, II, and III represent the different clusters of the tree. Pm3 paralog Pm3TD_RGL255 was used as an outgroup. 
note that another allele from Bat-Shlomo Pm3TDm is found at the other end of the tree. T. durum (cultivated tetraploid wheat) alleles were distributed more or less evenly between the T. aestivum and $T$. dicoccoides clusters. The branches of the tree were screened for signatures of episodic diversifying selection (Kosakovsky Pond et al. 2011). Most of the signatures were found in the T. aestivum cluster (Fig. 2). To investigate the spatial structure of the distribution of the alleles, a spatial principal component analysis (sPCA) was conducted followed by a global spatial structure test as implemented in "adegenet" $\mathrm{R}$ package (Jombart et al. 2008). sPCA is designed to incorporate a spatial component, the geographic relatedness between genotypes, into PCA, thus revealing patterns of population structure that are related to the spatial distribution of the genotypes that are not revealed by standard PCA (Jombart et al. 2008). This analysis revealed that there is a significant global spatial structure of the alleles $(P=0.008)$. The global structure means that alleles from distant origins are also genetically distant. The first spatial principal component vector (PC1) was superimposed on a map of Israel (Fig. 3). The map shows that alleles with $\mathrm{PC} 1=4$ are the most frequent $(44 \%)$ and are the majority of the alleles $(>70 \%)$ south of the lake of Galilee while, in the northern part of Israel, alleles from the low and the high ends of PC1 are scattered.

\section{$P m 3$ sequence variability.}

Variation analysis along the Pm3 genes of $T$. aestivum, $T$. durum, and $T$. dicoccoides detected 365 variable sites. The main difference between $T$. dicoccoides and T. aestivum alleles lies in LRR 19 to 24. This difference is a block of concentrated variable sites (Fig. 4). Another block of variable sites was ob-

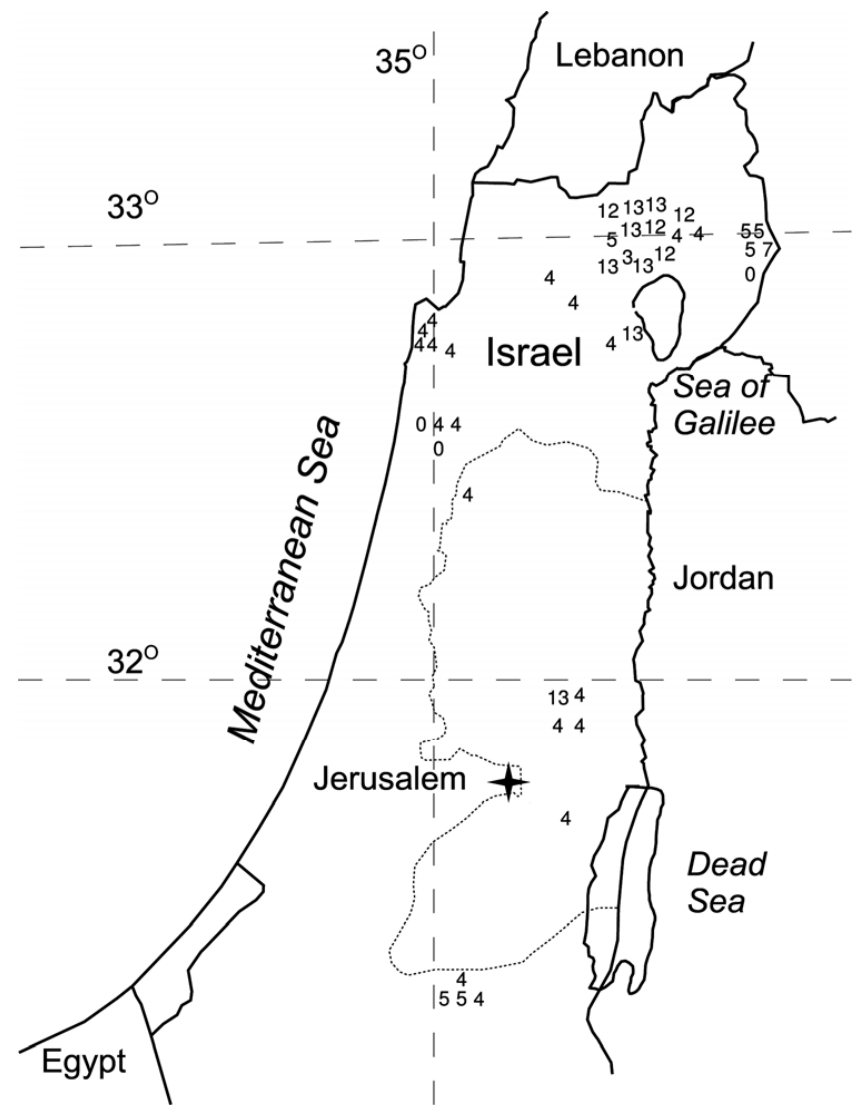

Fig. 3. First spatial principal component vector $(\mathrm{PC} 1)$ superimposed on the map of Israel. Each Pm3 sequence is represented by a number positioned at the site where it originated. The number is the rounded value of the sequence along PC1 of the spatial component analysis (sPCA) range 0 to 13. Overlapping numbers were slightly scattered. served in the internal spacer (IS). This IS block was present in several $T$. dicoccoides alleles as well as in several T. aestivum alleles. However, the IS blocks in the two species are not identical and, in each species, there are two haplotypes. All the blocks along the gene bear numerous nonsynonymous substitutions. It is interesting to note that the T. aestivum alleles differed from each other by blocks of mutations present in one or two alleles while the $T$. dicoccoides alleles differed from each other by single mutations scattered along the gene (Fig. 4). The most distinct functional alleles, $P m 3 a$ and $P m 3 b$, differ from other T. aestivum alleles mainly in the NBS domain, as described by Brunner and associates (2010). Analysis of positive selection by the fast unbiased Bayesian approximation (FUBAR) algorithm detected two codons under positive selection in the T. dicoccoides alignment and seven codons in the T. aestivum alignment (Table 1). The positively selected codons were all from the LRR domain. In six of them, more than one amino acid substitution was observed whereas, in the T. dicoccoides alignment, only one substitution per codon was observed. All the positively selected codons between LRR 21 and 28 were located within the solvent-exposed position of the LXXLXLXXN/C motif (Table 1). The BLOSUM62 (Henikoff and Henikoff 1992) substitution score was calculated for each of the pairs of residues in each of the seven codons. The minimum score of six of the positively selected codons in the $T$. aestivum population was negative. Recombination detection analysis (RDP) showed that gene conversion events among $P m 3$ alleles and between Pm3 alleles and their paralogs occurred in the diversity blocks (Supplementary Fig. S1).

\section{PM3-LRR repeat delineation.}

Delineation of repeats based on the most probable LxxLx LxxN/C motifs is shown in the supporting information (Supplementary Fig. S2). Interestingly, this sequence analysis revealed the existence of an additional repeat with respect to the previous PM3-LRR delineation (Yahiaoui et al. 2004) immediately downstream of repeat 22 and, therefore, designated herein as 22a. This repeat was ignored thus far due to its unusual composition $(\mathrm{AxxVxV})$, which only rarely occurs in LRR (e.g., in PFAM family LRR_5: sequences E6U637 and F3AP56). Quite similar to other plant LRR domains, PM3LRR repeats are highly variable, ranging from 19 (LRR 9) to 57 (LRR 24) amino acids, with an average length of approximately 26 amino acids (aa). The most obvious difference between the PM3 LRR domain and other R protein LRR domains is the sheer number of repeats (29 LRR). Eight of them are longer than 30 aa and two pairs of repeat duplicates occurred: LRR 6/7 and the unusually long repeats 12/13 (38 aa).

\section{Prediction of a 3D structure of the PM3 LRR domain.}

PM3 LRR template analysis indicates that the PM3-LRR repeats are highly similar to four fragments (f1 to f4) found in our LRR structural database (Slootweg et al. 2013). These fragments are the Internalin A from Listeria monocytogenes (PDB code: 1o6s [f1 and f2]) (Schubert et al. 2001), the Tolllike receptor 3 from Homo sapiens (PDB code: $2 \mathrm{a} 0 \mathrm{z}$ [f3]) (Bell et al. 2005), and YopM from Yersinia pestis (PDB code: $1 \mathrm{j} 15$ [f4]) (Evdokimov et al. 2001). Parameters describing the overall LRR geometry such as curvature, twist, out of planarity, and so on are very similar for all four templates. This further underpins the reliability of using the joint fragment remote homology modeling (JFRHM) procedure in this case. The optimal global LRR frame of the PM3-LRR was built starting from the above-mentioned four fragments (f1 to f4) of $4,3,13$, and 9 repeats, respectively. After repeated rounds of loop remodeling and side-chain optimization, the model was brought to a resolution of $2.8 \AA$ root mean square from an opti- 
mal polypeptide path, which reaches the general accepted limits of deterministic models derived from X-ray crystallography.

\section{Characterization of the LRR model.}

The LRR region of PM3 plays a critical role in determining PM3 specificity toward different fungal isolates (Brunner et al. 2010; Yahiaoui et al. 2006). In order to describe sequence to structure relations in this region, we mapped the amino acid diversity found within and between wild wheat $T$. dicoccoides and domesticated wheat $T$. aestivum to our 3D model of the PM3 LRR region.
Most PM3-LRR have a cysteine at position 9 that forms a stabilizing Cys ladder with side chains oriented toward the LRR core (Fig. 5A). In addition to this Cys ladder, another stabilizing pattern, based on phenylalanine (Phe) stacking, is observed in LRR 10,11, 12, 13, and 14 close to the N-terminal end of each LRR motif. The Phe-stack consists of F828, F858, F896, F934, and F962 (Fig. 5A). In all cases, these phenylalanines are followed by a proline which further reduces the local flexibility of the chain. Interestingly, this second LRR stabilizing pattern appears around the very long duplicates LRR 12 and 13 (38 aa), reinforcing the structure in that region. More-

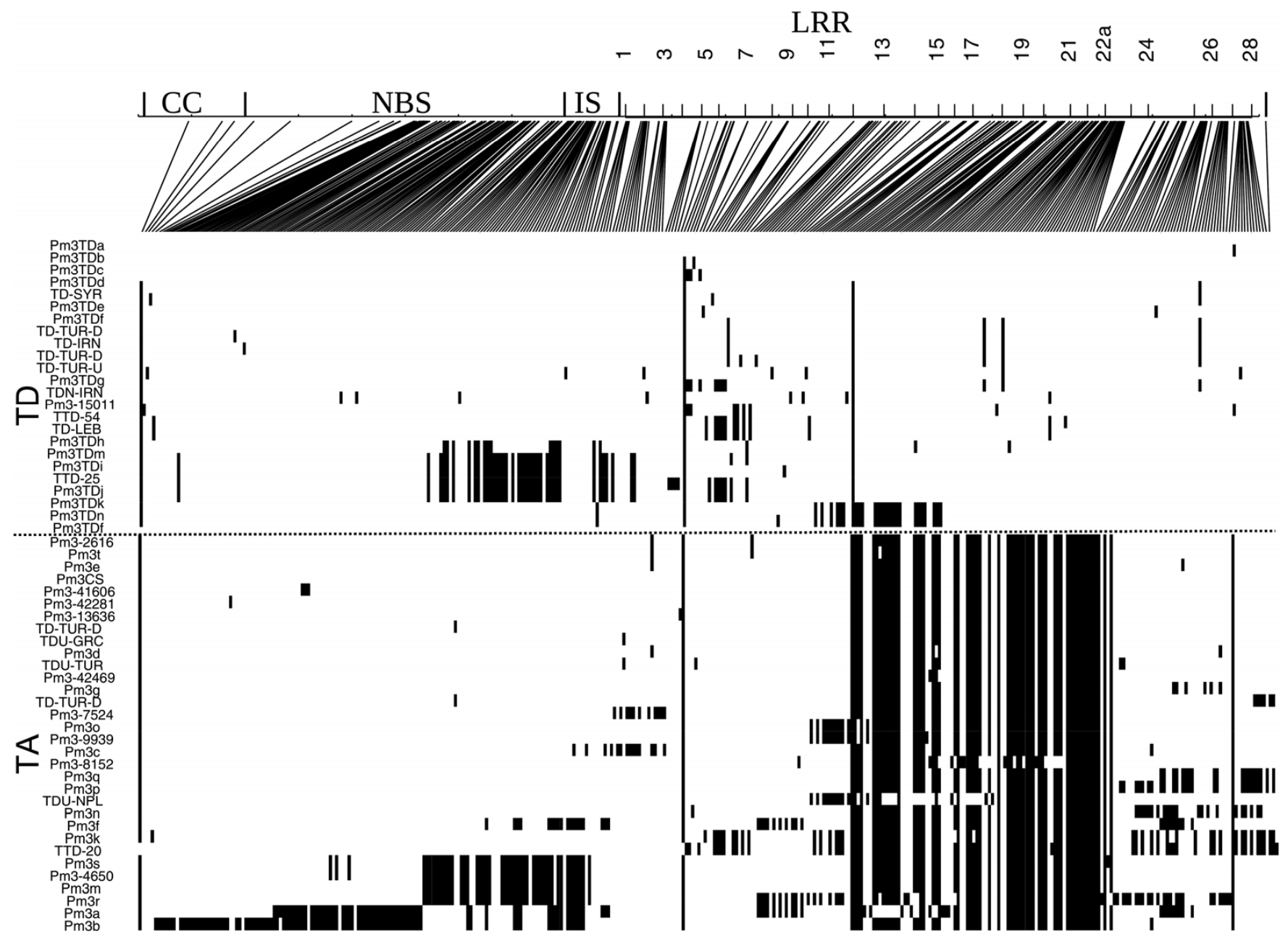

Fig. 4. Variation along Pm3 DNA sequences shown as a plot of variable sites along Pm3. Nucleotides that differ from the reference allele Pm3TDa are represented by short vertical black lines for each sequence. Pm3TDa was chosen as the reference allele because it was the most frequent sequence found in Triticum dicoccoides. It is the uppermost sequence in the figure. Only variable sites are represented in the plot. Top: the bar represents the Pm3 sequence. Protein domains are indicated above. Below the bar: lines connecting the variable site position in the gene to its position in the plot. Dashed line separates the T. aestivum sequences (TA) from the T. dicoccoides sequences (TD).

Table 1. Positivity selected codons in PM3 as inferred by fast unbiased Bayesian approximation (Delport et al. 2010)

\begin{tabular}{lccc}
\hline Species, position & Posterior probability & Min BLOSUM62 score & Residues in the position $^{\text {Codon within LXXLXLXX/C motif number }}$ \\
\hline Triticum aestivum & 0.988 & -2 & \\
1330 & 0.979 & 0 & S,F,I,A \\
1134 & 0.977 & -4 & R,E \\
1332 & 0.974 & -4 & W,D,Y,C,R \\
1155 & 0.969 & -1 & Y,G,H,L,R \\
1358 & 0.966 & -2 & T,R,K \\
1354 & 0.964 & -2 & S,Y,A \\
1153 & & & T,G,D \\
T. dicoccoides & 0.958 & -2 & G,H \\
1153 & 0.970 & -1 & A,R \\
849 & & 22 & 22 \\
\hline
\end{tabular}

\footnotetext{
${ }^{a}$ Asterisk (*) indicates residues are located in leucine-rich repeat 11 but not in the LXXLXLXX/C motif.
} 
over, due to their extreme length, these repeats would have been more difficult to accommodate within the space of a standard LRR repeat. Therefore, it is remarkable that the sequence has a high propensity for helix formation onto the side of the convex region of LRR 12 and 13. This adds up to the stabilization induced by the Cys ladder, which is located in the beginning of the LRR repeat, and the Phe-Pro stack, toward the end of the LRR repeat. At least two separate regions of clustered aro-
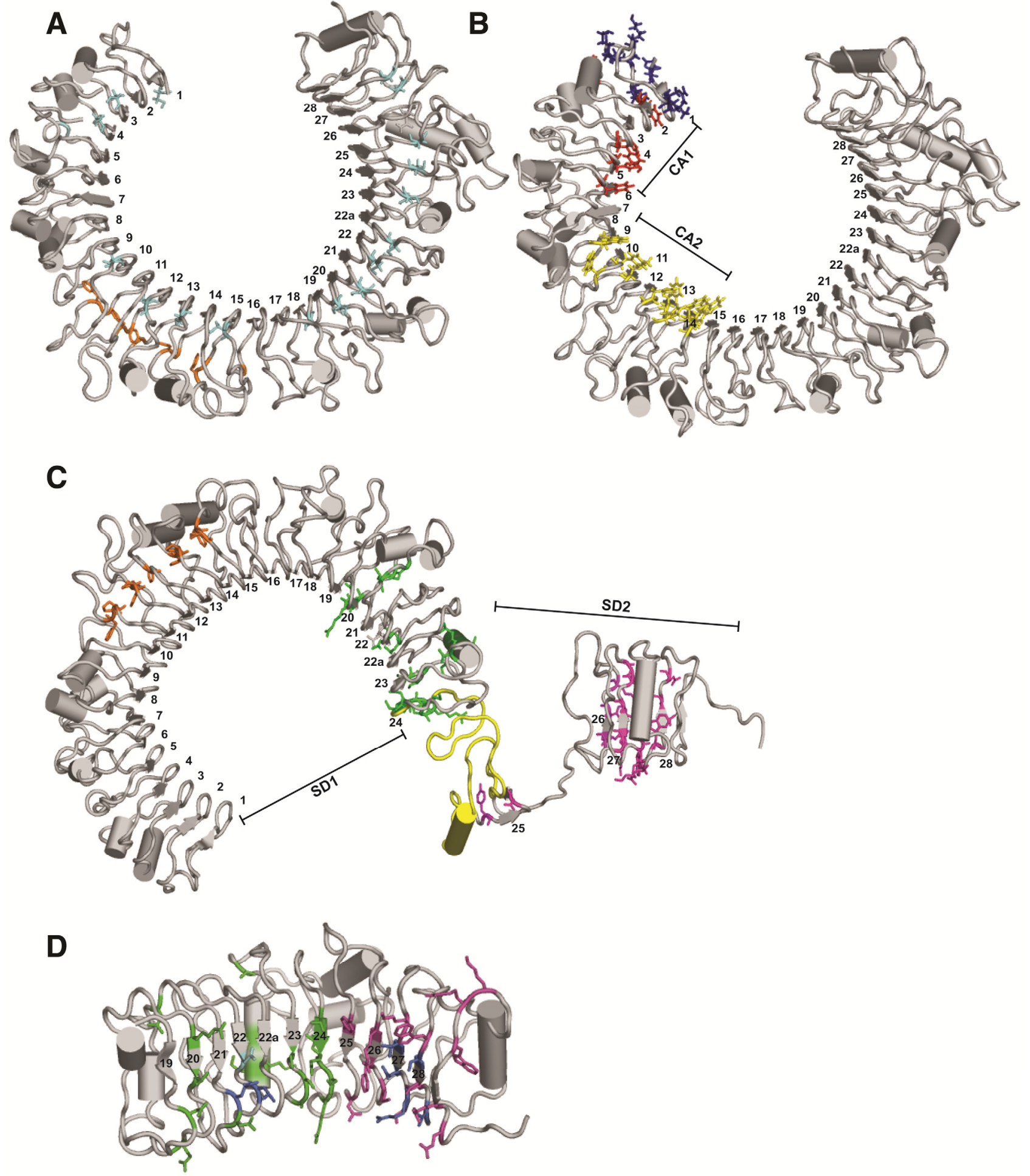

Fig. 5. Three-dimensional (3D) models of the PM3 leucine-rich repeat (LRR) domain created by joint fragment remote homology modeling. A, Continuous model of the PM3 LRR domain. LRR 24 is folded into the LRR backbone. Stabilizing elements of the PM3 LRR domain are cysteine residues that are depicted in cyan and a phenylalanine-proline ladder (orange). B, Continuous model of the PM3 LRR domain. LRR 24 is folded into the LRR backbone. Conserved putative interaction patches present on the surface of PM3-LRR are shown. The cluster of basic amino acids is colored in blue while the two aromatic amino acid patches CA1 and CA2 are colored in red and yellow, respectively. C, Discontinuous model of the PM3 LRR domain. The domain is divided into two subdomains: SD1 and SD2. Green residues represent sites of interspecies diversity. Residues depicted in purple show the variability within hexaploid wheat. LRR 24 is colored in yellow. Orange residues represents the phenylalanine-proline ladder. D, LRR 19 to 28 . Green residues represent sites of interspecies diversity. Residues depicted in purple show the variability hotspot within hexaploid wheat. Positive selection sites found in Triticum aestivum are depicted in blue. The positive selection site in LRR 22 that was found in T. dicoccoides and T. aestivum is shown in cyan. 
matic amino acids (CA1 and CA2) emerge in the N-terminal LRR: CA1 is formed by Y604, Y632, H651, Y653, H655, and F679 and CA2 is formed by W767, Y791, Y793, H808, H837, F864, H867, F902, W904, and Y905 (Fig. 5B). Additionally, a patch of positively charged amino acids is formed by H581, K584, R588, K595, K597, H600, H601, and R603 in the Nterminal region of PM3-LRR (Fig 5B).

Toward the C-terminal end of the PM3 LRR domain, there is a large repeat (LRR 24) that consists of 57 aa (versus an average length of 26 aa). Because LRR 24 and its neighboring repeats 23 and 25 show a cysteine at position 9, keeping the Cys ladder continuous, and LRR 23 and 25 are shorter on their convex side, which creates more space to accommodate LRR 24 within the horseshoe structure, LRR 23, 24, and 25 were modeled as regular repeats. It is worth noting that, in contrast to LRR 12 and 13, the sequence of repeat 24 shows a high propensity for flexibility and intrinsic disorder which may allow its convex part either to mold between LRR 23 and 25 or extend outside the regular horseshoe shape of the PM3 LRR. This suggests that two different conformations of the LRR domain are possible. To reflect this indeterminacy, two models were built: i) a continuous model in which LRR 24 integrates into the regular horseshoe structure (Fig. 5A and B) and ii) a discontinuous model in which a linker located between residues 1,237 and 1,278 divides the PM3 LRR into two subdomains, SD1 (LRR 1 to 24) and SD2 (LRR 25 to 28) (Fig. 5C). However, both models can be reconciled into a dynamic view in which the LRR domain adopts these two configurations at different stages in time, and this transition is caused by the folding or unfolding of repeat LRR 24.

It is even more intriguing that this unusual long repeat separates the region (LRR 19 to 24) that shows the highest variability between wild $T$. dicoccoides and domesticated hexaploid $T$. aestivum and the region (LRR 25 to 28, SD2) where the highest variability within the T. aestivum set occurs (Fig. 5C and D). This clear segregation of the variability pattern is echoed also by a slightly shifted localization of the two variability regions on the LRR surface. The interspecies polymorphisms in LRR 19 to 24 occur within the core motif LxxLxLxxN/C and in repeats 20, 22, and $22 \mathrm{a}$ also in the loop region (Fig. 5D). Therefore, amino acid polymorphisms are not only located on the concave part of the horseshoe but also toward the lateral side of the protein structure. The T. aestivum-specific polymorphism hotspot is more confined within the core motif at solvent-exposed positions and, hence, centered on the concave part of the horseshoe (Fig. 5D).

\section{DISCUSSION}

\section{Geographical distribution of $\mathrm{Pm} 3$ alleles.}

The phylogenetic tree constructed based on Pm3 alleles derived from tetraploid and hexaploid wheat species revealed that the Pm3 alleles derived from accessions of the $T$. dicoccoides judaicum race are different from the alleles of the $T$. $d i$ coccoides huranum race (Fig. 2). This is in agreement with genome-wide analyses that show that the judaicum race is well separated from the huranum race (Luo et al. 2007; Ozkan et al. 2002, 2005; Poyarkova 1988; Poyarkova et al. 1991; Sela et al. 2009, 2011). The sPCA analysis revealed that PC1 values of $P m 3$ alleles originating from north of the Lake of Galilee are from both extremes of PC1, while most of the alleles that originated south of the Lake of Galilee have low PC1 values (Fig. $3)$. Therefore, we can conclude that the center of diversity of Pm3 alleles in Israel overlaps with the center of genetic diversity previously detected in wild emmer populations (Nevo and Beiles 1989). This overlap suggests that Pm3 diversity within T. dicoccoides has evolved before the current distribution of the populations and indicates that some of the diversity patterns are stable and may be maintained by balancing selection.

\section{Different evolutionary history}

of $P m 3$ genes in wild and domesticated wheat.

The phylogenetic tree obtained in the current study clearly shows that the $T$. dicoccoides Pm3 alleles are distinct from the T. aestivum alleles. Surprisingly, T. aestivum alleles have undergone a rapid diversification after formation of hexaploid wheat, whereas the $T$. dicoccoides alleles remain less diverse. This higher diversity of T. aestivum Pm3 alleles is in contrast with the fact that genome-wide nucleotide diversity was reduced in $T$. aestivum by $70 \%$ compared with $T$. dicoccoides (Haudry et al. 2007; Ozkan et al. 2005).

We found that the evolution of T. aestivum Pm3 alleles was not driven solely by point mutations or indels but that gene conversion has played a major role in the evolution of the $T$. aestivum alleles, as was also suggested earlier (Bhullar et al. 2010; Srichumpa et al. 2005; Yahiaoui et al. 2006, 2009). In the current study, we were able to identify that paralogous genes took part in the gene conversion events, and to determine that these events are statistically significant. Therefore, the higher rate of evolution in T. aestivum Pm3 genes is facilitated by gene conversion events among homologs and paralogs generating new resistance specificities. Apparently, gene conversion events in the $T$. dicoccoides natural populations have less selective advantage, resulting in their complete elimination or erosion of their signatures.

The different evolutionary history of $P m 3$ genes in T. aestivum and $T$. dicoccoides is underscored by a distinct positive selection pattern. For Pm3 genes from T. aestivum, seven codons under positive selection were detected in the C-terminal LRR (LRR 21 to 28 ) region. All of them are located within the LXXLXLXXN/C motif and most of them with negative BLOSUM62 scores between the residues. Three of these codons are known to be involved in the determination of specificity (Brunner et al. 2010; Yahiaoui et al. 2006, 2009), indicating a coevolution between pathogen and host. In contrast, we found only two codons under positive selection in wild emmer wheat Pm3 genes. One of them is not located in the LRR motif (Table 1).

The lack of selective pressure on wild emmer Pm3 genes can be explained by the observation that $P m 3$ has high presence-or-absence variation in the natural populations. This is usually associated with low sequence diversity (Shen et al. 2006). The resistance tests have shown that many Pm3-negative genotypes are resistant, suggesting that wild emmer populations have other powdery mildew resistance genes to protect them (e.g., PmG16 and PmG3M) (Ben-David et al. 2010; Xie et al. 2012). Therefore, powdery mildew resistance diversity might be maintained by different genes and not by a Pm3allelic series. This may explain the low diversity found in $P m 3$ genes of T. dicoccoides as compared with $\mathrm{LrlO}$ in this species and resistance $(R)$ genes in other species (Ellis et al. 1999; Seeholzer et al. 2010; Sela et al. 2011).

\section{The LRR 3D model suggests differences in AVR recognition sites between $T$. aestivum and $T$. dicoccoides proteins.}

It is remarkable that the longest repeat (LRR 24) separates the protein region of highest interspecies diversity (LRR 19 to 24) from the region with the highest diversity within $T$. aestivum PM3 proteins (LRR 25 to 28). In this region (LRR 25 to 28), four of seven positively selected codons were detected. Furthermore, the importance of single amino acid changes in this region for recognition specificity was demonstrated by Brunner and associates (2010) and Yahiaoui and associates 
(2006). In contrast, in $T$. dicoccoides, the only positions where positive selection could be detected are LRR 11 and 22. These observations suggest that PM3 proteins of these two species recognize AVR proteins that might be quite different in amino acid composition or shape or that distinct detection mechanism are involved (see below). The AVR proteins AVR567 from flax rust interact directly with $\mathrm{L}$ proteins and were proposed to bind to $\mathrm{N}$ - and $\mathrm{C}$-terminal LRR between the two ends of the horseshoe structure (Wang et al. 2007). If the direct-interaction hypothesis for T. aestivum PM3 holds true, the N- and C-terminal LRR binding model could be possible also for the AVRPM3 interaction. Brunner and associates (2010) showed that polymorphic amino acids within LRR 1 (or the spacer region) and LRR 26 of PM3B and PM3C determine their recognition specificity. Based on the flexible structure of the PM3-LRR conformation, it is possible that, upon direct binding of AVRPM3 in T. aestivum, LRR 24 undergoes structural rearrangements resulting in the discontinuous conformation. This would destabilize the hypersensitive-response-repressing interaction between NBS and LRR (Takken et al. 2006), resulting in fast activation of resistance responses.

The allelic diversification in T. aestivum could be a result of a fast host-pathogen coevolution and direct interaction of AVR proteins with PM3 proteins or could be a consequence of a host range expansion of a small group of $B$. graminis $\mathrm{f}$. sp. tritici isolates. B. graminis f. sp. tritici isolates collected from wild $T$. dicoccoides can hardly infect $T$. aestivum plants (BenDavid et al. 2009). Therefore, the current B. graminis f. sp. tritici population that can infect $T$. aestivum had to go through a host expansion. This could have happened several times from different lineages followed by rapid evolution of the $B$. graminis f. sp. tritici population on the new host, as happened in Triticale (Troch et al. 2012). The rapid evolution of the pathogen may then be followed by rapid selection for new resistance or alleles. Furthermore, T. aestivum grows in various humid regions around the world where $B$. graminis f. $\mathrm{sp}$. tritici has good developing conditions while $T$. dicoccoides is restricted to more arid habitats. Therefore, $T$. aestivum may be infected by a more diverse panel of $B$. graminis $\mathrm{f}$. sp. tritici isolates.

In T. aestivum, Pm3 alleles are widespread in all agricultural areas worldwide and show a high degree of allelic differentiation (Bhullar et al. 2009, 2010; Kaur et al. 2008). In contrast, in wild $T$. dicoccoides populations, we see a high degree of presence and absence polymorphisms and low sequence diversity. This difference could be driven by interaction with effector or AVR proteins that are different from effectors in $B$. graminis f. sp. tritici collected from hexaploid wheat. Due to positive selection in LRR 11 and 21, we propose a model in which AVR proteins are suggested to bind to the central LRR part of $T$. dicoccoides PM3 proteins and PM3-mediated activation may not involve the structural rearrangement of LRR 24. This could be associated with an indirect recognition of AVR proteins, and the involvement of other $R$ genes present in $T$. $d i$ coccoides.

\section{Are island domains involved in AVR interaction?}

Longer interstrand regions are also called island domains and are found in many LRR-containing proteins (Matsushima and Miyashita 2012). With increased length, these regions often form loops that stretch outside the confined convex surface, making it less regular. For BRI (Kinoshita et al. 2005) and TLR5 (Yoon et al. 2012), it has been shown that these proteins bind their ligands through island domains and, in TLR5, this loop undergoes structural changes upon interaction with flagellin. PM3 proteins are hypothesized to bind their matching AVR proteins directly (Brunner et al. 2010; Yahiaoui et al. 2006). It will be interesting to test whether the island domains are involved in AVR-protein binding, especially in the loop regions of LRR 22a and 23, which are located in the region of interspecies sequence variability (LRR 19 to 24). Here, in addition to the polymorphisms in solvent-exposed residues, LRR 22a and 23 show numerous polymorphisms in the loop regions. To date, we can only speculate why $T$. aestivum and $T$. dicoccoides Pm3 genes have different amino acid requirements in these positions and whether these loop regions are required for differential AVR recognition. The importance of this region is underscored by the fact that the only site of positive selection that is common at the same position in T. aestivum and T. dicoccoides is located in LRR 22.

\section{Intermolecular interaction potential of the PM3 protein.}

The intermolecular interaction potential of a protein is highly dependent on the geometry and composition of the molecular surface in its various locations. Hence, the PM3-LRR $3 \mathrm{D}$ model can point to putative interaction hotspots in conserved and in variable regions. Interaction hotspots in conserved regions hint at obligate interactions resisting the natural mutation drift (e.g., regions involved in intradomain interactions) (Nooren and Thornton, 2003). Interaction hotspots in variable regions might indicate interfaces responsible for specific intermolecular interactions, such as those with various elicitors (Espadaler et al. 2005).

For example, the high density of conserved aromatic amino acids in the N-terminal part of the concave side of PM3-LRR might be instrumental for the intramolecular interaction potential with the coiled-coil and NBS domains. At least two separate patches-CA1 and CA2 - emerge in this region (Fig. 5B). Similarly a positively charged patch also builds up in the $\mathrm{N}$ terminal region of the PM3-LRR (Fig. 5B). It is important to note here that similar positive patches are present on the surface of Lr10-LRR (Sela et al. 2012) and Rx-LRR (Slootweg et al. 2013), which clearly indicates an obligate function for this patch, at least in this group of resistance proteins.

This study demonstrates the usefulness of the integration of an evolutionary analysis at the population level with modeling at the 3D molecular level to get insights about putative interactions of $\mathrm{R}$ proteins with their pathogen effectors. The results of the diversity or evolutionary study of $P m 3$ genes from the wild wheat southern population and domesticated wheat integrated with the 3D model of the PM3 LRR region which is the main determinant of recognition specificity gave us new insights into how PM3-mediated effector recognition might work. These results suggest that PM3 in T. dicoccoides and in T. aestivum differs in both effector recognition sites and evolutionary pathways, and that the two differences may be correlated. These interesting aspects provide starting points to further investigate the mode of recognition of PM3 proteins.

\section{MATERIALS AND METHODS}

\section{Plant material.}

In all, 208 accessions of $T$. dicoccoides were screened for the presence of a Pm3-like gene. Of these, 90 were from a new collection collected in 2009 from 72 sites across Israel and 118 accessions were from an old collection (1980s), collected from 12 sites in Israel (Nevo and Beiles 1989). All collections are maintained in the Institute of Evolution, University of Haifa, Israel. DNA was extracted from 10-day-old seedlings using the ArchivePure DNA extraction kit (5 Prime Inc., Gaithersburg, MD, U.S.A.). The screen for $P m 3$ presence was done using the STS polymerase chain reaction (PCR) marker indicative for Pm3, located upstream of the start codon, as described by Yahiaoui and associates (2006). Resistance tests were conducted using detached leaf assays, as described by Bhullar and 
associates (2009). Our wheat powdery mildew (B. graminis f. sp. tritici) isolates 98275, 97011, 96224, 96229, and 96244 originated from the former mildew collections of Agroscope Reckenholz-Tänikon ART.

\section{Isolation and sequencing of $P m 3$.}

The full-length $P m 3$ gene was isolated from accessions that showed an indication for the presence of $P m 3$ when tested with the Pm3-indicative STS marker. Two nested PCR reactions were used, as described previously by Srichumpa and associates (2005) and Yahiaoui and associates (2006). Reactions were modified to fit the standard protocol of Herculase II Fusion DNA Polymerase (Agilent Technologies, Santa Clara, CA, U.S.A.). PCR products were purified and sequenced using Big Dye Terminator chemistry on ABI 3700 or ABI 3730 instruments (Applied Biosystems, Foster City, CA, U.S.A.). Sequence reads were first analyzed and assembled using Phred, Phrap, and Consed software, provided by B. Ewing, P. Green, and D. Gordon, respectively. These sequences were then aligned using MUSCLE (Edgar 2004) and manually corrected using BioEdit (Hall 2007). Each SNP was visually rechecked on the chromatogram using Consed to ensure its quality. The obtained sequences were deposited in the National Center for Biotechnology Information GenBank under accession numbers JF739306 to JF739353. The diversity and phylogenetic analyses also included $P m 3$ sequences of $T$. dicoccoides, T. durum, and T. aestivum obtained from previous studies (Bhullar et al. 2009, 2010; Srichumpa et al. 2005; Yahiaoui et al. 2004, 2006, 2009). Because of the integration of Pm3 sequences obtained in different studies in addition to the new Pm3 sequences produced in the current study, we have kept the original nomenclature for each collection of sequences, as follows: Pm3TD followed by a lowercase letter represent new $T$. dicoccoides alleles obtained in the current study; TTD sequences are from $T$. dicoccoides accessions collected in Israel and contributed by M. Feldman (Yahiaoui et al. 2009); TD followed by letters indicates $T$. dicoccoides from Iran (IRN), Turkey Diyarbakir (TUR_D), Turkey Urfa (TUR_U), Syria (SYR), and Lebanon (LEB); TDU indicates T. durum from Greece (GRC), Turkey (TUR), or Nepal (NPL). Pm3 followed by a letter indicates functional $P m 3$ alleles. $P m 3$ followed by an accession number indicates a nonfunctional $T$. aestivum allele (Bhullar et al. 2009, 2010; Srichumpa et al. 2005; Yahiaoui et al. 2004, 2006, 2009). Paralogs from T. dicoccoides were named Pm3TD_RGL followed by the respective accession number.

\section{Statistical analysis.}

The maximum likelihood tree was constructed using MEGA (Tamura et al. 2007). The Pm3 paralog Pm3TD_RGL255 obtained in this study was chosen as an outgroup. A random effects branch-site model as implemented in the HYPHY package was used to detect branches in the tree subjected to episodic diversifying selection (Kosakovsky Pond et al. 2011). To test the spatial distribution of the alleles in relation to their genetic distance, sPCA was conducted followed by a global spatial structure test as implemented in "adegenet" $\mathrm{R}$ package (Jombart et al. 2008). Geographic coordinates of the collection sites that were used in the sPCA were recorded by GPS or inferred from a map. The geographic connection network between genotypes for the SPCA was calculated using the Delaunay triangulation algorithm implemented in chooseCN R function (adegenet package). Only the first global PC was retained for the global spatial structure test. The first PC was superimposed on a map of Israel (Fig. 3). FUBAR (Delport et al. 2010) was used to screen for codons under positive selection (i.e., codons with posterior probability above 0.95 that the ratio of nonsynonymous substitutions rate to synonymous sub- stitution rate is larger than $1[\mathrm{dN} / \mathrm{dS}>1])$. Recombination detection analysis was conducted using RDP (Martin et al. 2005a). In this software the combined results of the algorithms of RDP, GENECONV, MaxChi, BootScan, and SiScan were used to detect recombination events among $P m 3$ sequences and to detect events between $P m 3$ alleles and $P m 3$ paralogous RGL sequences. Events were considered significant when they were detected by three or more methods with $P<0.01$ after 1,000 permutations (Gibbs et al. 2000; Martin et al. 2005b; Padidam et al. 1999; Smith 1992). A variation plot was constructed using in-house R scripts (R Development Core Team 2011).

\section{PM3-LRR modeling.}

Secondary structure propensity was built as the consensus derived from predictions performed with SOPMA (Geourjon and Deleage 1995), GOR IV (Garnier et al. 1996), PsiPred (Jones 1999), Jpred (Cole et al. 2008), HNN (Guermeur 1997), and Prof (Ouali and King 2000). Linkers were predicted by DLP-SVM (Miyazaki et al. 2002). Intrinsic disorder profiles were raised using RONN (Yang et al. 2005) and DisEMBL (Linding et al. 2003).

Model building of the PM3-LRR followed the JFRHM (Sela et al. 2012; Slootweg et al. 2013). As a first step, we ranked putative LRR motifs based on a structural LRR motif database, their secondary structure prediction, and physico-chemical properties. In a second step, we generated the most probable repeat structure followed by the selection and joining of best local templates from the structural LRR database. Local optimization of target to template alignment was performed with SLIDE software for interactive threading (Hanganu et al. 2009). Modeling was performed with Insight II (Accelrys, San Diego, CA, U.S.A.). Accuracy of the model was evaluated with MetaMQAP (Pawlowski et al. 2008) and refined using CHARMm forcefield in NAMD (Phillips et al. 2005). Variability at a given site along the multiple alignments of PM3 protein sequences was defined as the average of the BLOSUM90 substitution matrix values between all sequences (Henikoff and Henikoff 1992). This was mapped onto the 3D structure using a Python script written for PyMol (DeLano Scientific LLC, Palo Alto, CA, U.S.A.).

\section{ACKNOWLEDGMENTS}

This work was supported by grants from the European Union sixth frame-work program (FP6) in the BioExploit project (number CT-2005513949), The Israel Science Foundation (ISF) grant number 205/08, and ISF equipment grants 1478/04, and 1719/08. L. N. Spiridon and A.-J. Petrescu acknowledge support from CNCSIS grant PN-II-ID-PCE-2011-30342 and from Romanian Academy projects 1 and 3 of IBAR. B. Keller and T. Jordan acknowledge support by Advanced Investigator Grant of the European Research Council (ERC-2009-AdG 249996, Durable resistance).

\section{LITERATURE CITED}

Bell, J. K., Botos, I., Hall, P. R., Askins, J., Shiloach, J., Segal, D. M., and Davies, D. R. 2005. The molecular structure of the Toll-like receptor 3 ligand-binding domain. Proc. Natl. Acad. Sci. U.S.A. 102:1097610980.

Ben-David, R., Peleg, Z., Saranga, Y., Dinoor, A., Korol, A., and Fahima, T. 2009. asymmetric reciprocal virulence pattern among Blumeria graminis isolates originating from domesticated wheat and its wild progenitor. In: 12th Int. Cereal Rusts Powdery Mildew Conf. Antalya, Turkey.

Ben-David, R., Xie, W., Peleg, Z., Saranga, Y., Dinoor, A., and Fahima, T. 2010. Identification and mapping of PmG16, a powdery mildew resistance gene derived from wild emmer wheat. Theor. Appl. Genet. 121:499-510.

Bhullar, N. K., Street, K., Mackay, M., Yahiaoui, N., and Keller, B. 2009. Unlocking wheat genetic resources for the molecular identification of 
previously undescribed functional alleles at the $P m 3$ resistance locus. Proc. Natl. Acad. Sci. U.S.A. 106:9519-9524.

Bhullar, N. K., Zhang, Z., Wicker, T., and Keller, B. 2010. Wheat gene bank accessions as a source of new alleles of the powdery mildew resistance gene Pm3: A large scale allele mining project. BMC Plant Biol. 10:88.

Brunner, S., Hurni, S., Streckeisen, P., Mayr, G., Albrecht, M., Yahiaoui, N., and Keller, B. 2010. Intragenic allele pyramiding combines different specificities of wheat Pm3 resistance alleles. Plant J. 64:433-445.

Cole, C., Barber, J. D., and Barton, G. J. 2008. The Jpred 3 secondary structure prediction server. Nucleic Acids Res. 36:W197-201.

Dangl, J. L., and Jones, J. D. G. 2001. Plant pathogens and integrated defence responses to infection. Nature 411:826-833.

Delport, W., Poon, A. F. Y., Frost, S. D. W., and Pond, S. L. K. 2010 Datamonkey 2010: A suite of phylogenetic analysis tools for evolutionary biology. Bioinformatics 26:2455-2457.

Dodds, P. N., Lawrence, G. L., and Ellis, J. G. 2001. Six amino acid changes confined to the leucine-rich repeat $\beta$-strand/ $\beta$-turn motif determine the difference between the P and P2 rust resistance specificities in flax. Plant Cell 13:163-178.

Dodds, P. N., Lawrence, G. J., Catanzariti, A. M., Teh, T., Wang, C. I. A., Ayliffe, M. A., Kobe, B., and Ellis, J. G. 2006. Direct protein interaction underlies gene-for-gene specificity and coevolution of the flax resistance genes and flax rust avirulence genes. Proc. Natl. Acad. Sci. U.S.A. 103:8888-8893.

Edgar, R. C. 2004. MUSCLE: Multiple sequence alignment with high accuracy and high throughput. Nucleic Acids Res. 32:1792-1797.

Ellis, J. G., Lawrence, G. J., Luck, J. E., and Dodds, P. N. 1999. Identification of regions in alleles of the flax rust resistance gene $\mathrm{L}$ that determine differences in gene-for-gene specificity. Plant Cell 11:495-506.

Espadaler, J., Romero-Isart, O., Jackson, R. M., and Oliva, B. 2005. Prediction of protein-protein interactions using distant conservation of sequence patterns and structure relationships. Bioinformatics 21:33603368.

Evdokimov, A. G., Anderson, D. E., Routzahn, K. M., and Waugh, D. S. 2001. Unusual molecular architecture of the Yersinia pestis cytotoxin YopM: A leucine-rich repeat protein with the shortest repeating unit. J. Mol. Biol. 312:807-821.

Garnier, J., Gibrat, J. F., and Robson, B. 1996. GOR method for predicting protein secondary structure from amino acid sequence. Comput. Methods Macromol. Sequence Anal. 266:540-553.

Geourjon, C., and Deleage, G. 1995. SOPMA: Significant improvements in protein secondary structure prediction by consensus prediction from multiple alignments. Comput. Appl. Biosci. 11:681-684.

Gibbs, M. J., Armstrong, J. S., and Gibbs, A. J. 2000. Sister-Scanning: A Monte Carlo procedure for assessing signals in recombinant sequences. Bioinformatics 16:573-582

Guermeur, Y. 1997. Combinaison de classifieurs statistiques, application à la prédiction de la structure secondaire des protéines. University of Paris, Paris.

Hall, T. 2007. BioEdit Version 7.0. 9. Computer Program and Documentation. Ibis Biosciences, Carlsbad, CA, U.S.A.

Hanganu, A., Micluta, M., Popa, B., Spiridon, L., and Tacutu, R. 2009 "Slide": An interactive threading refinement tool for homology modeling. Romanian J. Biochem. Cell Biol. 46:123-127.

Haudry, A., Cenci, A., Ravel, C., Bataillon, T., Brunel, D., Poncet, C., Hochu, I., Poirier, S., Santoni, S., Glemin, S., and David, J. 2007. Grinding up wheat: A massive loss of nucleotide diversity since domestication. Mol. Biol. Evol. 24:1506-1517.

Henikoff, S., and Henikoff, J. G. 1992. Amino-acid substitution matrices from protein blocks. Proc. Natl. Acad. Sci. U.S.A. 89:10915-10919.

Jombart, T., Devillard S., Dufour, A. B., and Pontier, D. 2008. Revealing cryptic spatial patterns in genetic variability by a new multivariate method. Heredity 110:92-103.

Jones, D. T. 1999. Protein secondary structure prediction based on position-specific scoring matrices. J. Mol. Biol. 292:195-202.

Kanzaki, H., Yoshida, K., Saitoh, H., Fujisaki, K., Hirabuchi, A., Alaux, L., Fournier, E., Tharreau, D., and Terauchi, R. 2012. Arms race co-evolution of Magnaporthe oryzae AVR-Pik and rice Pik genes driven by their physical interactions. Plant J. 72:894-907.

Kaur, N., Street, K., Mackay, M., Yahiaoui, N., and Keller, B. 2008. Molecular approaches for characterization and use of natural disease resistance in wheat. Eur. J. Plant Pathol. 121:387-397.

Kinoshita, T., Cano-Delgado, A. C., Seto, H., Hiranuma, S., Fujioka, S., Yoshida, S., and Chory, J. 2005. Binding of brassinosteroids to the extracellular domain of plant receptor kinase BRI1. Nature 433:167171

Kosakovsky Pond, S. L., Murrell, B., Fourment, M., Frost, S. D. W., Delport, W., and Scheffler, K. 2011. A random effects branch-site model for detecting episodic diversifying selection. Mol. Biol. Evol. 28:3033-3043.
Krasileva, K. V., Dahlbeck, D., and Staskawicz, B. J. 2010. Activation of an Arabidopsis resistance protein is specified by the in planta association of its leucine-rich repeat domain with the cognate oomycete effector. Plant Cell 22:2444-2458

Linding, R., Jensen, L. J., Diella, F., Bork, P., Gibson, T. J., and Russell, R. B. 2003. Protein disorder prediction: Implications for structural proteomics. Structure 11:1453-1459.

Luo, M. C., Yang, Z. L., You, F. M., Kawahara, T., Waines, J. G., and Dvorak, J. 2007. The structure of wild and domesticated emmer wheat populations, gene flow between them, and the site of emmer domestication. Theor. Appl. Genet. 114:947-959.

Martin, D. P., Posada, D., Crandall, K. A., and Williamson, C. 2005a. A modified bootscan algorithm for automated identification of recombinant sequences and recombination breakpoints. AIDS Res. Hum. Retroviruses 21:98-102.

Martin, D. P., Williamson, C., and Posada, D. 2005b. RDP2: Recombination detection and analysis from sequence alignments. Bioinformatics 21:260-262.

Matsushima, N., and Miyashita, H. 2012. Leucine-rich repeat (LRR) domains containing intervening motifs in plants. Biomolecules 2:288-311.

Miyazaki, S., Kuroda, Y., and Yokoyama, S. 2002. Characterization and prediction of linker sequences of multi-domain proteins by a neural network. J. Struct. Funct. Genomics 2:37-51.

Nevo, E., and Beiles, A. 1989. Genetic Diversity of wild emmer wheat in Israel and Turkey-structure, evolution, and application in breeding. Theor. Appl. Genet. 77:421-455.

Nevo, E., Korol, A. B., Beiles, A., and Fahima, T. 2002. Evolution of Wild Emmer Wheat Improvement: Population Genetics, Genetic Resources, and Genome Organization of the Wheat's Progenitor, Triticum dicoccoides. Springer, New York.

Nooren, I. M. A., and Thornton, J. M. 2003. Structural characterisation and functional significance of transient protein-protein interactions. J. Mol. Biol. 325:991-1018

Ouali, M., and King, R. D. 2000. Cascaded multiple classifiers for secondary structure prediction. Protein Sci. 9:1162-1176.

Ozkan, H., Brandolini, A., Schafer-Pregl, R., and Salamini, F. 2002. AFLP analysis of a collection of tetraploid wheats indicates the origin of emmer and hard wheat domestication in southeast Turkey. Mol. Biol. Evol. 19:1797-1801.

Ozkan, H., Brandolini, A., Pozzi, C., Effgen, S., Wunder, J., and Salamini, F. 2005. A reconsideration of the domestication geography of tetraploid wheats. Theor. Appl. Genet. 110:1052-1060.

Padidam, M., Sawyer, S., and Fauquet, C. M. 1999. Possible emergence of new geminiviruses by frequent recombination. Virology 265:218-225.

Pawlowski, M., Gajda, M. J., Matlak, R., and Bujnicki, J. M. 2008. MetaMQAP: A meta-server for the quality assessment of protein models. BMC Bioinf. 9:403.

Peleg, Z., Saranga, Y., Krugman, T., Abbo, S., Nevo, E., and Fahima, T. 2008. Allelic diversity associated with aridity gradient in wild emmer wheat populations. Plant Cell Environ. 31:39-49.

Phillips, J. C., Braun, R., Wang, W., Gumbart, J., Tajkhorshid, E., Villa, E., Chipot, C., Skeel, R. D., Kale, L., and Schulten, K. 2005. Scalable molecular dynamics with NAMD. J. Comput. Chem. 26:1781-1802.

Poyarkova, H. 1988. Morphology, geography and infraspecific taxonomics of Triticum dicoccoides Körn-a retrospective of 80 years of research. Euphytica 38:11-23.

Poyarkova, H., Gerechteramitai, Z. K., and Genizi, A. 1991. 2 Variants of wild emmer (Triticum dicoccoides) native to Israel-morphology and distribution. Can. J. Bot. Rev. Can. Bot. 69:2772-2789.

R Development Core Team. 2011. R: A Language and Environment for Statistical Computing. The R Foundation for Statistical Computing, Vienna.

Schubert, W. D., Gobel, G., Diepholz, M., Darji, A., Kloer, D., Hain, T., Chakraborty, T., Wehland, J., Domann, E., and Heinz, D. W. 2001 Internalins from the human pathogen Listeria monocytogenes combine three distinct folds into a contiguous internalin domain. J. Mol. Biol. 312:783-794

Seeholzer, S., Tsuchimatsu, T., Jordan, T., Bieri, S., Pajonk, S., Yang, W., Jahoor, A., Shimizu, K. K., Keller, B., and Schulze-Lefert, P. 2010. Diversity at the Mla powdery mildew resistance locus from cultivated barley reveals sites of positive selection. Mol. Plant-Microbe Interact. 23:497-509.

Sela, H., Cheng, J. P., Jun, Y., Nevo, E., and Fahima, T. 2009. Divergent diversity patterns of NBS and LRR domains of resistance gene analogs in wild emmer wheat populations. Genome 52:557-565.

Sela, H., Loutre, C., Keller, B., Schulman, A., Nevo, E., Korol, A., and Fahima, T. 2011. Rapid linkage disequilibrium decay in the Lr10 gene in wild emmer wheat (Triticum dicoccoides) populations. Theor. Appl. Genet. 122:175-187.

Sela, H., Spiridon, L. N., Petrescu, A. J., Akerman, M., Mandel-Gutfreund, 
Y., Nevo, E., Loutre, C., Keller, B., Schulman, A. H., and Fahima, T. 2012. Ancient diversity of splicing motifs and protein surfaces in the wild emmer wheat (Triticum dicoccoides) LR10 coiled coil (CC) and leucine-rich repeat (LRR) domains. Mol. Plant Pathol. 13:276-287.

Shen, J. D., Araki, H., Chen, L. L., Chen, J. Q., and Tian, D. C. 2006. Unique evolutionary mechanism in R-genes under the presence/absence polymorphism in Arabidopsis thaliana. Genetics 172:1243-1250.

Slootweg, E. J., Spiridon, L. N., Roosien, J., Butterbach, P., Pomp, R., Westerhof, L., Wilbers, R., Bakker, E., Bakker, J., Petrescu, A.-J., Smant, G., and Goverse, A. 2013. Structural determinants at the interface of the ARC2 and LRR domains control the activation of the NB-LRR plant immune receptors Rx1 and Gpa2. Plant Physiol. 162:1510-1528.

Smith, J. M. 1992. Analyzing the mosaic structure of genes. J. Mol. Evol. 34:126-129.

Srichumpa, P., Brunner, S., Keller, B., and Yahiaoui, N. 2005. Allelic series of four powdery mildew resistance genes at the Pm3 locus in hexaploid bread wheat. Plant Physiol. 139:885-895.

Takken, F. L. W., and Tameling, W. I. L. 2009. To nibble at plant resistance proteins. Science 324:744-746.

Takken, F. L. W., Albrecht, M., and Tameling, W. I. L. 2006. Resistance proteins: Molecular switches of plant defence. Curr. Opin. Plant Biol. 9:383-390.

Tamura, K., Dudley, J., Nei, M., and Kumar, S. 2007. MEGA4: Molecular evolutionary genetics analysis (MEGA) software version 4.0. Mol. Biol. Evol. 24:1596-1599.

Troch, V., Audenaert, K., Bekaert, B., Hofte, M., and Haesaert, G. 2012. Phylogeography and virulence structure of the powdery mildew population on its 'new' host triticale. BMC Evol. Biol. 12:76.

Wang, C. I. A., Guncar, G., Forwood, J. K., Teh, T., Catanzariti, A. M. Lawrence, G. J., Loughlin, F. E., Mackay, J. P., Schirra, H. J., Anderson, P. A., Ellis, J. G., Dodds, P. N., and Kobe, B. 2007. Crystal structures of flax rust avirulence proteins AvrL567-A and -D reveal details of the structural basis for flax disease resistance specificity. Plant Cell 19:2898 2912.

Wei, T., Gong, J., Jamitzky, F., Heckl, W. M., Stark, R. W., and Rossle, S. C. 2008. LRRML: A conformational database and an XML description of leucine-rich repeats (LRR). BMC Struct. Biol. 8:47.

Xie, W., Ben-David, R., Zeng, B., Distelfeld, A., Roder, M. S., Dinoor, A., and Fahima, T. 2012. Identification and characterization of a novel powdery mildew resistance gene PmG3M derived from wild emmer wheat, Triticum dicoccoides. Theor. Appl. Genet. 124:911-922.

Yahiaoui, N., Srichumpa, P., Dudler, R., and Keller, B. 2004. Genome analysis at different ploidy levels allows cloning of the powdery mildew resistance gene $P m 3 b$ from hexaploid wheat. Plant J. 37:528-538.

Yahiaoui, N., Brunner, S., and Keller, B. 2006. Rapid generation of new powdery mildew resistance genes after wheat domestication. Plant $\mathrm{J}$. 47:85-98.

Yahiaoui, N., Kaur, N., and Keller, B. 2009. Independent evolution of functional $P m 3$ resistance genes in wild tetraploid wheat and domesticated bread wheat. Plant J. 57:846-856.

Yang, Z. R., Thomson, R., McNeil, P., and Esnouf, R. M. 2005. RONN: The bio-basis function neural network technique applied to the detection of natively disordered regions in proteins. Bioinformatics 21:33693376

Yoon, S. I., Kurnasov, O., Natarajan, V., Hong, M. S., Gudkov, A. V., Osterman, A. L., and Wilson, I. A. 2012. Structural basis of TLR5-flagellin recognition and signaling. Science 335:859-864.

\section{AUTHOR-RECOMMENDED INTERNET RESOURCES}

¿HyPhy - Hypothesis testing using Phylogenies: www.hyphy.org

University of Washington Genome Sciences Department Phred, Phrap, and Consed software page: www.phrap.org 